\title{
On a covering problem in the hypercube
}

\author{
Lale Özkahya \\ email: ozkahya@illinoisalumni.org
}

\author{
Brendon Stanton \\ The Citadel \\ Department of Mathematics \\ Charleston, SC 29409 USA \\ email: bstanton@citadel.edu
}

October 23, 2018

\begin{abstract}
In this paper, we address a particular variation of the Turán problem for the hypercube. Alon, Krech and Szabó (2007) asked "In an $n$-dimensional hypercube, $Q_{n}$, and for $\ell<d<n$, what is the size of a smallest set, $S$, of $Q_{\ell}$ 's so that every $Q_{d}$ contains at least one member of $S$ ?" Likewise, they asked a similar Ramsey type question: "What is the largest number of colors that we can use to color the copies of $Q_{\ell}$ in $Q_{n}$ such that each $Q_{d}$ contains a $Q_{\ell}$ of each color?" We give upper and lower bounds for each of these questions and provide constructions of the set $S$ above for some specific cases.
\end{abstract}

\section{Introduction}

For graphs $Q$ and $P$, let $\operatorname{ex}(Q, P)$ denote the generalized Turán number, i.e., the maximum number of edges in a $P$-free subgraph of $Q$. The $n$-dimensional hypercube, $Q_{n}$, is the graph whose vertex set is $\{0,1\}^{n}$ and whose edge set is the set of pairs that differ in exactly one coordinate. For a graph $G$, we use $n(G)$ and $e(G)$ to denote the number of vertices and the number of edges of $G$, respectively.

In 1984, Erdős [8] conjectured that

$$
\lim _{n \rightarrow \infty} \frac{\operatorname{ex}\left(Q_{n}, C_{4}\right)}{e\left(Q_{n}\right)}=\frac{1}{2}
$$

Note that this limit exists, because the function above is non-increasing for $n$ and bounded. The best upper bound $\operatorname{ex}\left(Q_{n}, C_{4}\right) / e\left(Q_{n}\right) \leq 0.62256$ was obtained by Thomason and Wagner [16] by slightly improving the bound 0.62284 given by Chung [4]. Brass, Harborth and Nienborg [3] showed that the lower bound is $\frac{1}{2}(1+1 / \sqrt{n})$, when $n=4^{r}$ for integer $r$, and $\frac{1}{2}(1+0.9 / \sqrt{n})$, when $n \geq 9$. 
Erdös [8] also asked whether $o\left(e\left(Q_{n}\right)\right)$ edges in a subgraph of $Q_{n}$ would be sufficient for the existence of a cycle $C_{2 k}$ for $k>2$. The value of $\operatorname{ex}\left(Q_{n}, C_{6}\right) / e\left(Q_{n}\right)$ is between $1 / 3$ and 0.3941 given by Conder [6] and $\mathrm{Lu}$ [11], respectively. On the other hand, nothing is known for the cycle of length 10. Except $C_{10}$, the question of Erdös is answered positively by showing that $\operatorname{ex}\left(Q_{n}, C_{2 k}\right)=o\left(e\left(Q_{n}\right)\right)$ for $k \geq 4$ in [4], [7] and [10].

A generalization of Erdős' conjecture above is the problem of determining $\operatorname{ex}\left(Q_{n}, Q_{d}\right)$ for $d \geq 3$. As for $d=2$, the exact value of $\operatorname{ex}\left(Q_{n}, Q_{3}\right)$ is still not known. The best lower bound for $\operatorname{ex}\left(Q_{n}, Q_{3}\right) / e\left(Q_{n}\right)$ has been $1-(5 / 8)^{0.25} \approx 0.11086$ due to Graham, Harary, Livingston and Stout [14 until recently Offner [12] improved it to 0.1165. The best upper bound is $\operatorname{ex}\left(Q_{n}, Q_{3}\right) / e\left(Q_{n}\right) \leq 0.25$ due to Alon, Krech and Szabó [1]. They also gave the best bounds for $\operatorname{ex}\left(Q_{n}, Q_{d}\right), d \geq 4$, as

$$
\Omega\left(\frac{\log d}{d 2^{d}}\right)=1-\frac{\operatorname{ex}\left(Q_{n}, Q_{d}\right)}{e\left(Q_{n}\right)} \leq \frac{\frac{4}{(d+1)^{2}}}{\frac{4}{d(d+2)}} \quad \text { if } d \text { is odd } \text { is even. }
$$

These Turán problems are also asked when vertices are removed instead of edges and most of these problems are also still open. In a very recent paper, Bollobás, Leader and Malvenuto [2] discuss open problems on the vertex-version and their relation to Turán problems on hypergraphs.

Here, we present results on a similar dual version of the hypercube Turán problem that is asked by Alon, Krech and Szabó in [1]. Let $\mathcal{H}_{n}^{i}$ denote the collection of $Q_{i}$ 's in $Q_{n}$ for $1 \leq i \leq n-1$. Call a subset of $\mathcal{H}_{n}^{\ell}$ a $(d, \ell)$-covering set if each member of $\mathcal{H}_{n}^{d}$ contains some member of this set, i.e., $\mathcal{H}_{n}^{d}$ is covered by this set. A smallest $(d, \ell)$-covering set is called optimal. Alon, Krech and Szabó [1] asked what the size of the optimal $(d, \ell)$-covering set of $Q_{n}$ is for fixed $\ell<d$. Call this function $f^{(\ell)}(n, d)$. Determining this function when $\ell=1$ is equivalent to the determination of $\operatorname{ex}\left(Q_{n}, Q_{d}\right)$, since $\operatorname{ex}\left(Q_{n}, Q_{d}\right)+f^{(1)}(n, d)=e\left(Q_{n}\right)$ and the best bounds for $f^{(1)}(n, d)$ are given in [1] as (1). In [1, also the Ramsey version of this problem is asked as follows. A coloring of $\mathcal{H}_{n}^{\ell}$ is $d, \ell$-polychromatic if all colors appear on each copy $Q_{d}$ 's. Let $p c^{(\ell)}(n, d)$ be the largest number of colors for which there exists a $d, \ell$-polychromatic coloring of $\mathcal{H}_{n}^{\ell}$.

Let $c^{(\ell)}(n, d)$ be the ratio of $f^{(\ell)}(n, d)$ to the size of $\mathcal{H}_{n}^{\ell}$, i.e.,

$$
c^{(\ell)}(n, d)=\frac{f^{(\ell)}(n, d)}{2^{n-\ell}\left(\begin{array}{l}
n \\
\ell
\end{array}\right)} .
$$

One can observe that

$$
c^{(\ell)}(n, d) \leq \frac{1}{p c^{(\ell)}(n, d)},
$$


since any color class used in a $d, \ell$-polychromatic coloring is a $(d, \ell)$-covering set of $Q_{n}$. Note that the following limits exist, since $c^{(\ell)}(n, d)$ is non-decreasing, $p c^{(\ell)}(n, d)$ is non-increasing and both are bounded.

$$
c_{d}^{(\ell)}=\lim _{n \rightarrow \infty} c^{(\ell)}(n, d), \quad p_{d}^{(\ell)}=\lim _{n \rightarrow \infty} p c^{(\ell)}(n, d) .
$$

In Section 2, we obtain bounds on the polychromatic number.

Theorem 1. For integers $n>d>\ell$, let $0<r \leq \ell+1$ such that $r=d+1(\bmod \ell+1)$. Then

$$
e^{\ell+1}\left(\frac{d+1}{\ell+1}\right)^{\ell+1} \geq\left(\begin{array}{l}
d+1 \\
\ell+1
\end{array}\right) \geq p_{d}^{(\ell)} \geq\left\lceil\frac{d+1}{\ell+1}\right\rceil^{r}\left\lfloor\frac{d+1}{\ell+1}\right\rfloor^{\ell+1-r} \approx\left(\frac{d+1}{\ell+1}\right)^{\ell+1} .
$$

In Section 3, we present the following bounds on $c_{d}^{(\ell)}$ and $c^{(\ell)}(n, d)$.

Theorem 2. For integers $n>d>\ell$ and $r=d-\ell(\bmod \ell+1)$,

$$
\left(2^{d-\ell}\left(\begin{array}{l}
d \\
\ell
\end{array}\right)\right)^{-1} \leq c_{d}^{(\ell)} \leq\left\lceil\frac{d+1}{\ell+1}\right\rceil^{-r}\left\lfloor\frac{d+1}{\ell+1}\right\rfloor^{-(\ell+1-r)} .
$$

The determination of the exact values of $p_{d}^{\ell}$ and $c_{d}^{\ell}$ remains open. The lower and upper bounds on $c^{(\ell)}(n, d)$ provided in Theorem 2 and Theorem 3. respectively, are a constant factor of each other when $d$ and $\ell$ have a bounded difference from $n$.

Theorem 3. Let $n-d$ and $n-\ell$ be fixed finite integers, where $d>\ell$. Then, for sufficiently large $n$,

$$
c^{(\ell)}(n, d) \leq\left\lceil\frac{r \log (n-\ell)}{\log \left(\frac{r^{r}}{r^{r}-r !}\right)}\right\rceil \frac{1+o(1)}{2^{d-\ell}\left(\begin{array}{l}
d \\
l
\end{array}\right)},
$$

where $r=n-d$.

Finally, we show an exact result for $c^{(\ell)}(n, d)$ when $d=n-1$.

Theorem 4. For integers $n-1>\ell$,

$$
c^{(\ell)}(n, n-1)=\frac{\left\lceil\frac{2 n}{n-\ell}\right\rceil}{2^{n-\ell}\left(\begin{array}{c}
n \\
\ell
\end{array}\right)} .
$$

In our proofs, we make use of the following terminology. The collection of $i$-subsets of $[n]=\{1, \ldots, n\}, 1 \leq i \leq n$, is denoted by $\left(\begin{array}{c}{[n]} \\ i\end{array}\right)$. For an edge $e \in E\left(Q_{n}\right)$, $\operatorname{star}(e)$ denotes the coordinate that is different at endpoints of $e$. The set of coordinates whose values are 0 (or 1 , resp.) at both endpoints of $e$ are denoted by zero(e) (or one(e), resp.). For a subcube $F \subset Q_{n}$, $\operatorname{star}(F):=\cup_{e \subseteq E(F)} \operatorname{star}(e)$, one $(F):=\cap_{e \subseteq E(F)}$ one $(e)$ and $\operatorname{zero}(F):=\cap_{e \subseteq E(F)} \operatorname{zero}(e)$. Note that $E_{1}$ covers $E_{2}$ for $E_{1} \in \mathcal{H}_{n}^{\ell}$ and $E_{2} \in \mathcal{H}_{n}^{d}(d>\ell)$ if and only if $\operatorname{zero}\left(E_{2}\right) \subset \operatorname{zero}\left(E_{1}\right)$ and one $\left(E_{2}\right) \subset$ one $\left(E_{1}\right)$. 
Definition 5. For any $Q \in \mathcal{H}_{n}^{\ell}$ and $\operatorname{star}(Q)$ with coordinates $s_{1}<s_{2}<\ldots<s_{\ell}$, we define an $(\ell+1)$-tuple $w(Q)=\left(w_{1}, w_{2}, \ldots, w_{\ell+1}\right)$ as

- $w_{1}=\mid\left\{x \in\right.$ one $\left.(Q): x<s_{1}\right\} \mid$,

- $w_{j}=\left|\left\{x \in \operatorname{one}(Q): s_{j-1}<x<s_{j}\right\}\right|$, for $2 \leq j \leq \ell$,

- $w_{\ell+1}=\left|\left\{x \in \operatorname{one}(Q): x>s_{\ell}\right\}\right|$.

\section{Polychromatic Coloring of Subcubes}

Proof of Theorem 1. The lower bound:

For any $Q \in \mathcal{H}_{n}^{\ell}$ with $w(Q)=\left(w_{1}, w_{2}, \ldots, w_{\ell+1}\right)$, we define the color of each $Q \in \mathcal{H}_{n}^{\ell}$ as the $(\ell+1)$-tuple $c(Q)=\left(c_{1}, \ldots, c_{\ell+1}\right)$ such that

$$
\begin{array}{cll}
c_{i}=w_{i} & (\bmod k) & \text { if } 1 \leq i \leq r \text { and } \\
c_{i}=w_{i} & \left(\bmod k^{\prime}\right) & \text { if } r+1 \leq i \leq \ell+1,
\end{array}
$$

where $k=\lceil(d+1) /(\ell+1)\rceil$ and $k^{\prime}=\lfloor(d+1) /(\ell+1)\rfloor$. We show that this coloring is $d, \ell$ polychromatic.

Let $C \in \mathcal{H}_{n}^{d}$, where $\operatorname{star}(C)$ consists of the coordinates $a_{1}<a_{2}<\cdots<a_{d}$. We choose a color $\left(c_{1}, \ldots, c_{\ell+1}\right)$ arbitrarily and show that $C$ contains a copy of $Q_{\ell}$, call it $Q$, with this color.

Since $Q$ must be a subgraph of $C, \operatorname{zero}(C) \subset \operatorname{zero}(Q)$ and one $(C) \subset$ one $(Q)$. We define $\operatorname{star}(Q)=\left\{s_{1}, \ldots, s_{\ell}\right\}$ such that

$$
s_{i}= \begin{cases}a_{i k} & \text { if } 1 \leq i \leq r, \\ a_{r k+(i-r) k^{\prime}} & \text { if } r+1 \leq i \leq \ell .\end{cases}
$$

We include the remaining $d-\ell$ positions of $\operatorname{star}(C)$ to one $(Q)$ or $\operatorname{zero}(Q)$ such that $w(Q)=$ $\left(w_{1}, w_{2}, \ldots, w_{\ell+1}\right)$ satisfies (44). This is possible since by the definition of $r$, we have $d-\ell=$ $r(k-1)+(\ell+1-r)\left(k^{\prime}-1\right)$.

The upper bound:

Since $p c^{(\ell)}(n, d)$ is a non-increasing function of $n$, we provide an upper bound for this function when $n$ is sufficiently large which is also an upper bound for $p_{d}^{(\ell)}$.

For a subset $S$ of $[n]$, we define cube $(S)$ as the subcube $Q$ of $Q_{n}$ such that $\operatorname{star}(Q)=S$ and $\operatorname{zero}(Q)=[n] \backslash S$. Let $\mathcal{G}$ be a subfamily of $\mathcal{H}_{n}^{d}$ such that $\mathcal{G}=\left\{\operatorname{cube}(S): S \in\left(\begin{array}{c}{[n]} \\ d\end{array}\right)\right\}$. We define a coloring of the members of $\mathcal{G}$ as follows.

Consider a $d, \ell$-polychromatic coloring of $\mathcal{H}_{n}^{\ell}$ using $p$ colors, call this coloring $P$. Fix an arbitrary ordering of the copies of $Q_{\ell}$ 's in $Q_{d}$. We define a coloring of $\mathcal{H}_{n}^{d}$ such that the color of a copy of $Q_{d}$ is the list of colors of each $Q_{\ell}$ under $P$ in this fixed order. By using this coloring on the members of $\mathcal{G}$, we obtain a coloring of $\mathcal{G}$ using $p^{\left(\begin{array}{c}d \\ \ell\end{array}\right) 2^{d-\ell}}$ colors. 
Now, consider the auxiliary $d$-uniform hypergraph $\mathcal{G}^{\prime}$ whose vertex set is the set of coordinates $[n]$ and whose edge set is defined as the collection of $\operatorname{star}(E)$ 's for each $E$ in $\mathcal{G}$, i.e., $\mathcal{G}^{\prime}$ is a complete $d$-uniform hypergraph on the vertex set $[n]$. Also we define a coloring of the edges of $\mathcal{G}^{\prime}$ by using the colors on the corresponding members of $\mathcal{G}$ as described above. Ramsey's theorem on hypergraphs implies that there is a sufficiently large value of $n$ such that there exists a complete monochromatic subgraph on $d^{2}+d-1$ vertices in any edge coloring of $\mathcal{G}^{\prime}$ with $p^{\left(\begin{array}{c}d \\ \ell\end{array}\right) 2^{d-\ell}}$ colors. Let $K \subset[n]$ be the vertex set of a monochromatic complete subgraph of $\mathcal{G}^{\prime}$ on $d^{2}+d-1$ vertices. We define $S$ as the collection of $i d^{\text {th }}$ coordinates in $K, 1 \leq i \leq d$, so that there are at least $d-1$ coordinates between elements of $S$.

Claim 6. If $Q$ is a copy of $Q_{\ell}$ in cube $(S)$, then the color of $Q$ under $P$ depends only on $w(Q)$.

Proof. Let $E_{1}$ and $E_{2}$ be two different copies of $Q_{\ell}$ in cube $(S)$ such that $w\left(E_{1}\right)=w\left(E_{2}\right)$ according to Definition 5. There exists a subset $S^{\prime} \subset K$ with $\left|S^{\prime}\right|=d$ such that

- $\left(\operatorname{one}\left(E_{2}\right) \cup \operatorname{star}\left(E_{2}\right)\right) \subset S^{\prime}$, i.e., $E_{2}$ is contained in cube $\left(S^{\prime}\right)$ and

- the restriction of $E_{2}$ on $S^{\prime}$ gives the same vector as the restriction of $E_{1}$ on $S$.

Clearly, one can find $S^{\prime}$ that satisfies the first condition. It is also possible that $S^{\prime}$ fulfills the second condition, since we can remove or add up to $d-1$ coordinates from $K$ between consecutive coordinates of ones and stars in $E_{2}$ to define $S^{\prime}$. This implies that the colors of $E_{1}$ and $E_{2}$ are the same under $P$, since cube $(S)$ and cube $\left(S^{\prime}\right)$ have the same colors.

Hence, the number of colors used in any $d, \ell$-polychromatic coloring of $\mathcal{H}_{n}^{\ell}$ is at most the number of possible vectors $w(Q)$ for any $Q \in \mathcal{H}_{n}^{\ell}$. The number of possible $(\ell+1)$-tuples $w(Q)$ for any $Q \in \mathcal{G}$ is given by the number of partitions of at most $d-\ell$ ones into $\ell+1$ parts and therefore it is at most $\left(\begin{array}{c}d+1 \\ \ell+1\end{array}\right)$.

\section{The Covering Problem}

Proof of Theorem 2, Note that a trivial lower bound on $f^{(\ell)}(n, d)$ is given by the ratio of $\left|\mathcal{H}_{n}^{d}\right|$ to the exact number of $Q_{d}$ 's that a single $Q_{\ell}$ covers in $Q_{n}$. Thus, by (2), for all $n$,

$$
c^{(\ell)}(n, d) \geq\left\lceil\frac{2^{n-d}\left(\begin{array}{l}
n \\
d
\end{array}\right)}{\left(\begin{array}{l}
n-\ell \\
n-d
\end{array}\right)}\right\rceil \cdot \frac{1}{2^{n-\ell}\left(\begin{array}{l}
n \\
\ell
\end{array}\right)} .
$$

By using the equality $\left(\begin{array}{l}n \\ d\end{array}\right)\left(\begin{array}{c}d \\ d-\ell\end{array}\right)=\left(\begin{array}{l}n \\ \ell\end{array}\right)\left(\begin{array}{c}n-\ell \\ d-\ell\end{array}\right)$, we are done.

The upper bound is implied together by (3) and Theorem 1 .

We define a $(0,1)$-labelling of a set as an assignment of labels 0 or 1 to its elements.

Observation 7. Since any subcube $Q \subset Q_{n}$ is defined by $\operatorname{zero}(Q)$ and one $(Q)$, a $(d, \ell)$-covering set of $Q_{n}$ can be defined as a collection of $(0,1)$-labellings of sets chosen from $\left(\begin{array}{c}{[n]} \\ n-\ell\end{array}\right)$ such that any $(0,1)$-labelling of sets in $\left(\begin{array}{c}{[n]} \\ n-d\end{array}\right)$ is contained in at least one of the labelled $(n-\ell)$-sets. 
When providing constructions for the upper bounds in Theorems 3 and 4 , we provide constructions for the equivalent covering problem in Observation 7 .

\section{Proof of Theorem 3 ,}

We construct a $(d, \ell)$-covering of $Q_{n}$ by providing a construction for the equivalent problem as stated in Observation 7 . In the following, we describe this construction in two steps. First, we choose the $(n-\ell)$-subsets of $[n]$ to label and then, we describe an efficient way to $(0,1)$-label these sets.

Step 1: We make use of the following well-known result on the general covering problem. An $(n, k, t)$-covering is defined as a collection of $k$-subsets of $n$ elements such that every $t$-set is contained in at least one $k$-set. Let $C(n, k, t)$ be the minimum number of $k$-sets in an $(n, k, t)$ covering. Rödl proved the following result by also settling a long-standing conjecture of Erdös and Hanani [9]. For any fixed integers $k$ and $t$ with $2 \leq t<k<n$,

$$
\lim _{n \rightarrow \infty} \frac{C(n, k, t)}{\left(\begin{array}{l}
n \\
t
\end{array}\right) /\left(\begin{array}{l}
k \\
t
\end{array}\right)}=1
$$

By our assumption, $n-d$ and $n-\ell$ are fixed integers where $n-d<n-\ell$. By (6), there exists a $(n, n-\ell, n-d)$-covering $\mathcal{F}$ for sufficiently large $n$ such that $|\mathcal{F}|=(1+o(1))\left(\begin{array}{c}n \\ n-d\end{array}\right) /\left(\begin{array}{c}n-\ell \\ n-d\end{array}\right)$.

Step 2: We obtain a collection of $(0,1)$-labellings for each edge $e \in \mathcal{F}$ so that all $(0,1)$ labellings of $(n-d)$-subsets of $e$ are covered. The union of these $(0,1)$-labellings is a covering set.

An $r$-cut of an $r$-uniform hypergraph is obtained by partitioning its vertex set into $r$ parts and taking all edges that meet every part in exactly one vertex. An r-cut cover of a hypergraph is a collection of $r$-cuts such that each edge is in at least one of the cuts. An upper bound on the minimum size of an $r$-cut cover is shown by Cioabă, Kündgen, Timmons and Vysotsky in [5] using a probabilistic proof.

Theorem 8 ([5]). For every $r$, an r-uniform complete hypergraph on $n$ vertices can be covered with $\lceil c \log n\rceil r$-cuts if

$$
c>\frac{-r}{\log \left(\frac{r^{r}-r !}{r^{r}}\right)} .
$$

For a fixed edge $e$ of $\mathcal{F}$, let $\mathcal{G}_{e}$ be the complete $(n-d)$-uniform hypergraph on the vertex set of $e$. Let $C=\lceil c \log (n-\ell)\rceil$ be the size of a minimum $(n-d)$-cut cover of $\mathcal{G}_{e}$ as given by Theorem 8 . We obtain a collection of $(0,1)$-labellings of $e$ by labelling each cut in this cover such that the vertices in each part are labelled identically with 0 or 1 . Thus, the total number of $(0,1)$-labellings of $e$ is $2^{n-d} C$. (If some labelling of an edge is used more than once, then we count this labelling only once.) Finally, we use similarly labellings for each edge of $\mathcal{F}$ in the covering set. This yields that

$$
c^{(\ell)}(n, d) \leq \frac{1}{2^{n-\ell}\left(\begin{array}{c}
n \\
\ell
\end{array}\right)}\left(C(1+o(1)) 2^{n-d} \frac{\left(\begin{array}{c}
n \\
n-d
\end{array}\right)}{\left(\begin{array}{c}
n-\ell \\
n-d
\end{array}\right)}=C(1+o(1)) \frac{1}{2^{d-\ell}\left(\begin{array}{l}
d \\
l
\end{array}\right)},\right.
$$


where the last equality is obtained by using the relation $\left(\begin{array}{l}n \\ d\end{array}\right)\left(\begin{array}{c}d \\ d-\ell\end{array}\right)=\left(\begin{array}{l}n \\ \ell\end{array}\right)\left(\begin{array}{c}n-\ell \\ d-\ell\end{array}\right)$.

\section{Proof of Theorem 4,}

The lower bound follows from (5).

For the upper bound, we construct a collection of $(0,1)$-labellings of sets chosen from $\left(\begin{array}{c}{[n]} \\ n-\ell\end{array}\right)$, where singletons in $[n]$ have both 0 and 1 in some labelling. Let $k=\lceil n /(n-\ell)\rceil$. We choose a partition $[n]=\left(P_{1}, \ldots, P_{k}\right)$ such that $\left|P_{i}\right|=n-\ell$ for $i<k$. Let $P \in\left(\begin{array}{c}{[n]} \\ n-\ell\end{array}\right)$ such that $P_{k} \subset P$. In the covering set, we include two labellings of each of $P_{1}, \ldots, P_{k-1}, P$, where all labels are the same, either 0 or 1 .

\section{References}

[1] N. Alon, A. Krech and T. Szabó. Turán's theorem in the hypercube. SIAM J. Discrete Math., 21: 66-72, 2007.

[2] B. Bollobás, I. Leader and C. Malvenuto. Daisies and other Turán Problems. Combin., Probab. and Comp., 20: 743-747, 2011.

[3] P. Brass , H. Harborth and H. Nienborg. On the Maximum number of edges in a $C_{4}$-free subgraph of $Q_{n}$. J. Graph Theory, 19: 17-23, 1995.

[4] F. Chung. Subgraphs of a hypercube containing no small even cycles. J. Graph Theory, 16: 273-286, 1992.

[5] S. M. Cioabă, A. Kündgen, C. M. Timmons and V. V. Vysotsky. Covering complete rgraphs with spanning complete $r$-partite r-graphs. Combin., Probab. and Comput., 20: 519-527, 2011.

[6] M. Conder. Hexagon-free subgraphs of hypercubes. J. Graph Theory, 17: 477-479, 1993.

[7] D. Conlon. An extremal theorem in the hypercube. Electron. J. Combin., 17: R111, 2010.

[8] P. Erdös. On some problems in graph theory combinatorial analysis and combinatorial number theory. Graph Theory and Combinatorics, 1-17, 1984.

[9] P. Erdős and H. Hanani. On a limit theorem in combinatorial analysis. Publ. Math. Debrecen, 10: 10-13, 1963.

[10] Z. Füredi, L. Özkahya. On even-cycle-free subgraphs of the hypercube. J. of Combin. Theo., Ser. A, 118: 1816-1819, 2011. 
[11] Linyuan Lu. Hexagon-free subgraphs in hypercube $Q_{n}$. Private communication.

[12] D. Offner. Some Turán type results on the hypercube. Discrete Math., 102: 2905-2912, 2011.

[13] D. Offner. Polychromatic colorings of the hypercube. SIAM J. Discrete Math., 22: 450-454, 2008 .

[14] N. Graham, F. Harary, M. Livingston and Q. Stout. Subcube fault tolerance in hypercubes. Inform. and Comput., 102: 280-314, 1993.

[15] V. Rödl. On a packing and covering problem. European J. Combin., 6: 69-78, 1985.

[16] A. Thomason and P. Wagner. Bounding the size of square-free subgraphs of the hypercube. Discrete Math., 309: 1730-1735, 2009. 